\title{
The neuroprotective effects of progesterone on traumatic brain injury: current status and future prospects
}

\author{
Jing $\mathrm{WEI}^{1}$, Guo-min XIAO ${ }^{2, *}$ \\ ${ }^{1}$ Medical Center, The Affiliated Hospital of Hangzhou Normal University, Hangzhou 310015, China; ${ }^{2}$ Neurosurgery, The Affiliated \\ Hospital of Hangzhou Normal University, Hangzhou 310015, China
}

\begin{abstract}
Traumatic brain injury is the leading cause of morbidity and mortality in young adults. The secondary injury in traumatic brain injury consists of a complex cascade of processes that simultaneously react to the primary injury to the brain. This cascade has been the target of numerous therapeutic agents investigated over the last 30 years, but no neuroprotective treatment option is currently available that improve neurological outcome after traumatic brain injury. Progesterone has long been considered merely a female reproductive hormone. Numerous studies, however, show that progesterone has substantial pleiotropic properties as a neuroprotective agent in both animal models and humans. Here, we review the increasing evidence that progesterone can act as a neuroprotective agent to treat traumatic brain injury and the mechanisms underlying these effects. Additionally, we discuss the current progress of clinical studies on the application of progesterone in the treatment of traumatic brain injuries.
\end{abstract}

Keywords: progesterone; traumatic brain injury; neuroprotection; hormone

Acta Pharmacologica Sinica (2013) 34: 1485-1490; doi: 10.1038/aps.2013.160; published online 18 Nov 2013

\section{Introduction}

Traumatic brain injury (TBI) is one of the leading causes of injury-related death and severe disability worldwide, resulting in large direct and indirect costs to society. While the clinical management of TBI has been greatly improved with the development of standardized approaches to care, no medical treatment adjuncts have been proven to be effective in reducing mortality or limiting disability following $\mathrm{TBI}^{[1]}$. Progesterone (PROG) has long been considered merely a female reproductive hormone with little role in neuroprotection after brain injury. In fact, PROG is a neurosteroid that has many complex properties affecting the mechanisms involved in neuroprotection and repair after various types of brain injury ${ }^{[2]}$.

\section{Overview of neuroprotective agents in TBI}

TBI results in both primary and secondary injuries. Primary injuries are those injuries that immediately result from the initial impact or insult. In contrast, a secondary brain injury is a delayed response that results from a complex group of cellular and molecular responses to the primary injury. These are

\footnotetext{
* To whom correspondence should be addressed.

E-mail gxiao@hznu.edu.cn

Received 2013-06-24 Accepted 2013-09-28
}

characterized by a complex cascade of molecular and biochemical events that lead to neuro-inflammation, brain edema, and delayed neuronal death. Both primary and secondary injuries can ultimately result in cell death and irreversible damage.

In recent decades, our understanding of the cellular and molecular changes that occur after TBI has significantly increased. A number of new potential therapeutic targets have been identified which may enable the prevention of the onset or a reduction in the extent of secondary brain injuries. Currently, the mainstay of therapy for TBI is the removal of hematomas and the repair of significant skull fractures, along with supportive therapies aimed at maintaining perfusion and oxygenation to tissues. For patients who do not have operable lesions, the control of ICP ( $<20 \mathrm{mmHg})$, cerebral perfusion pressure (CPP, >60 mmHg) and systemic, and perhaps local, oxygenation are the cornerstones of intensive care unit management. Other necessary medical interventions are commonly employed, such as early enteral nutrition, maintenance of fluid volume, and prevention and treatment of complications, such as hyperthermia, hypernatremia, seizures, pneumonia, venous thromboembolism and stress-related mucosal bleeding ${ }^{[3]}$. Many of these therapies can influence the outcome for a TBI patient.

Because the primary injury is considered irreversible, the 
major opportunity for interventions that could prevent further neurological decline is in reversing or preventing secondary injuries. After decades of research, a number of experimental TBI models have been developed to study the mechanisms that occur following trauma, and many potential therapeutic interventions have been developed. In these preclinical studies, numerous pharmacological treatments have been identified, with at least 30 compounds and therapeutic interventions being the subject of more than 50 clinical trials over the last three decades. However, despite the encouraging preclinical results, none of these investigations have lead to any considerable improvement in the clinical outcome $\mathrm{e}^{[4]}$.

Most of the promising therapeutic strategies derived from experimental animal studies have failed when they are translated into the clinical setting of TBI. Glucocorticoids, once a mainstay of TBI treatment, are now known to be harmful ${ }^{[5,6]}$. The trial of magnesium sulfate was stopped when it was found that the magnesium sulfate treatment group had a significantly higher mortality rate than the control group ${ }^{[7]}$. More recently, citicoline, an endogenous substance offering potential neuroprotective properties that facilitates neurorepair after injury, was also shown to not improve the clinical function and cognitive status of patients after injury ${ }^{[8]}$.

The underlying reason for this disappointing failure likely varies from case to case. First, most injury models used to simulate TBI only represent the relatively homogenous injury types, while in reality, TBI is quite heterogenous. Clinical trials usually include a range of injury severities, whereas preclinical studies only use well-defined, highly controlled animal models of preselected severity. These conceptual and methodological issues should contribute to the difficulty in translating promising preclinical findings into human application.

Second, a detailed description of the therapeutic window, the pharmacokinetics, and the pharmacodynamics for each agent is not always available. Most animal studies use either pretreatment or very early treatment protocols, and such early intervention is not always possible in clinical TBI. Another popular problem for preclinical studies is the lack of pharmacokinetic or pharmacodynamic profiles of the drugs being examined or the levels of the drugs in the brain in relation to its therapeutic actions ${ }^{[0]}$.

Third, drug dosing schedules usually differ between preclinical and clinical trials, with the latter frequently using lower doses (to avoid potential toxicity) combined with more frequent dosages (eg, continuous infusions) that have not been supported by the preclinical data.

Finally, another major issue is that most of these preclinical studies have focused on a single component of a complex TBI cascade, which neglects the complexity and diversity of secondary injury mechanisms. To address this issue, therapeutic strategies that target multiple delayed injury factors by either combining agents that have complementary effects or using multipotential drugs that modulate multiple injury mechanisms should be considered. Thus, the simultaneous targeting of several injury factors using pleiotropic drugs may maximize the likelihood of developing a successful therapeutic interven- tion to improve the outcome of TBI patients. This recognition has led to the recent emphasis on pleiotropic neuroprotection.

\section{Preclinical studies of PROG in TBI}

It is now clear that biological sex alters the incidence and outcome of ischemia and TBI. Stroke risk increases with age in both sexes, and there is broad evidence that the outcome of an ischemic event is worse in older women than in their male counterparts. This sexually dimorphic disease pattern remains apparent in women well beyond their menopausal years ${ }^{[10,11]}$. In most animal experimental reports, the females tend to recover better than males following $\mathrm{TBI}^{[12,13]}$. The serendipitous discovery that gender and menstrual cycle may have an effect on an animal's response to experimental TBI ultimately led to the development of PROG as a neuroprotective agent.

PROG is a steroid hormone that is well known for its role in the menstrual cycle. It is produced by the ovaries and the placenta in females, as well as by the brain, and it plays a critical role in neuronal development during gestation. In the brains of males and females, PROG is synthesized by oligodendrocytes and some neurons in roughly equal amounts ${ }^{[14,15]}$. PROG receptors are widely expressed in the developing and adult brain, so various brain regions are the normal targets of PROG ${ }^{[16]}$. Indeed, the 10-fold increase in PROG synthesis during fetal growth is considered an evolutionary mechanism of PROG to protect the fetus during gestation. Because many processes involved in CNS repair after brain injury are thought to recapitulate what occurs during normal brain development ${ }^{[17]}$, PROG may be actively involved in TBI recovery.

After three decades of extensive research on the use of PROG in TBI, it is clear that PROG is a neurosteroid that affects multiple mechanisms involved in neuroprotection and repairation after various types of brain injury ${ }^{[2,17,18]}$. There are currently more than 200 articles reporting the neuroprotective effects of PROG. PROG has been tested in four species, including humans, and in 22 different injury models, including TBI, stroke, spinal cord injury and neurodegenerative disorders. Overall, all the findings from the various studies show that PROG infusion results in reduced neuronal loss, enhanced remyelination, improved functional recovery, and an overall decrease in cerebral edema ${ }^{[2]}$.

\section{Clinical studies of PROG in TBI}

Consistent with these promising preclinical studies, the first clinical study further supported the promise of PROG in the treatment of $\mathrm{TBI}^{[19]}$. This clinical research study was a single-center, open clinical trial that recruited 56 acute severe TBI patients (Glasgow Coma Scale $\leq 8$ ). Patients in the treatment group were given PROG via intramuscular injection at $1.0 \mathrm{mg} / \mathrm{kg}$ per $12 \mathrm{~h}$ for 5 consecutive days. The neurological outcomes of the patients were assessed using the Glasgow Outcome Scale (GOS), the verbal and motor functions scale and the Karnofsky Performance Scale (KPS). Follow-up assessment at 3 months found that the GOS, verbal functions and KPS in the PROG treatment group were more improved 
than those in the control group. These results indicated that successive early application of PROG would be beneficial in the treatment of TBI patients. This clinical trial, which was performed by a Chinese hospital, had certain weaknesses, such as a small number of patients, the single-centered, open trial design, and the lack of long-term follow up. Despite these issues, it is the first publication of a clinical study evaluating PROG in the treatment of TBI patients.

Subsequently, two randomized, double-blind, placebocontrolled phase II clinical trials for PROG have been conducted $^{[20,21]}$. Though distinct differences were noted between these two clinical trials, such as inclusion criteria, dosage of progesterone, administration route, and the duration of follow up, both studies have shown decreases in mortality following PROG treatment, as well as improvements in functional outcome after severe injury (Table 1). The two studies also confirmed that the drug is safe and tolerated by the head injury patients. In Wright's ProTECT II clinical trial, compared to the controls, PROG treatment produced a 50\% reduction in mortality at $30 \mathrm{~d}$ (rate ratio $0.43 ; 95 \%$ confidence interval 0.18 to 0.99$)$. The improvement reflected by the Disability Rating Scale (DRS) outcome at 30 days was even more encouraging for patients with moderate injuries $(P<0.02)^{[21]}$. Wright's findings were confirmed by Xiao's clinical trial ${ }^{[20]}$. In Xiao's study, it was found that PROG treatment significantly improved survival, as well as functional outcomes, at both 3 and 6 months. At 3 months, $47 \%$ of the PROG treated patients displayed better GOS functional outcomes compared to the $31 \%$ of the placebo patients $(P=0.034)$. At 6 months, the number was $58 \%$ for the PROG group compared to the $42 \%$ of the placebo group $(P=0.048)$. The 6 month mortality rate for the PROG group was $18 \%$, whereas it was $32 \%$ for the placebo group $(P=0.039)$.

\section{Mechanisms underlying PROG's neuroprotective effects}

PROG possesses pleiotropic effects that may markedly attenuate the injury cascade associated with TBI. Investigation into the cellular and biochemical pathways affected by PROG provided reasonable explanations for its efficacy in mitigating the effects of neuronal injury. Microarray analysis discovered that
PROG influences the expression of approximately 500 genes involved in regulating inflammation, apoptosis and vascular remodeling, which support the pleiotropic properties of PROG as a hormone ${ }^{[22]}$. Additionally, PROG acts on multiple proteins and receptors, making it a candidate for potent therapies.

\section{Edema}

PROG has been reported to reduce both vasogenic and cytotoxic edema after $\mathrm{TBI}^{[23,24]}$. It was found that the serum levels of PROG were inversely correlated with the degree of TBIinduced edema ${ }^{[25]}$. The underlying mechanisms by which PROG reduced the edema have not been fully elucidated, although several possible mechanisms have been proposed. PROG may decrease the levels of aquaporin 4 (AQP4), a water channel membrane protein that can, in turn, modulate cerebral edema ${ }^{[26]}$, inhibit active ion uptake through the $\mathrm{Na}^{+} / \mathrm{K}^{+}$ATPase and vessel growth associated with leaky blood- brain barrier function after TBI, modulate the vasopressin level, and reduce neurogenic inflammation ${ }^{[27]}$. All of these responses can help reduce cerebral edema after TBI and can lead to better functional outcomes ${ }^{[28]}$.

\section{Lipid peroxidation and oxidative stress}

There is evidence that PROG can reduce lipid peroxidation through the inhibition of free radical formation and by enhancing scavenger efficiency to more vigorously eliminate reactive oxygen species ${ }^{[29]}$. This anti-oxidative stress effect is achieved by upregulating antioxidant enzymes, such as superoxide dismutase (SOD) $)^{[30]}$, and by increasing the levels of mitochondrial glutathione, a critical free radical scavenger ${ }^{[31]}$. Reducing the presence of reactive oxygen and nitrogen species can help maintain membrane integrity and help stabilize the blood-brain barrier (BBB), which in turn improves cell survival following brain injury.

\section{Inflammation}

A number of studies have reported that PROG inhibits inflammation by modulating cytokine release and by inhibiting immune cell activation and migration ${ }^{[32-34]}$. PROG can reduce

Table 1. Two independently conducted, randomized, double-blind, placebo-controlled phase II clinical trials used to assess the efficacy of progesterone in TBI patients.

\begin{tabular}{lll}
\hline \multicolumn{1}{c}{ Characteristics } & \multicolumn{1}{c}{ Wright et al ${ }^{[21]}$} & Xiao et al ${ }^{[20]}$ \\
\hline Country & US & China \\
Glasgow coma scale (GCS) & 4 to 12 & $\leq 8$ \\
Time after injury & $<11 \mathrm{~h}$ & $<8 \mathrm{~h}$ \\
Delivery method (length) & Intravenous (3 d) & Intramuscular (5 d) \\
Outcome assessment post-injury & $30 \mathrm{~d}$ & 3 and 6 months \\
Randomization (progesterone:placebo) & $4: 1$ & $1: 1$ \\
Patients & 100 & 159 \\
Progesterone & 77 & 77 \\
Placebo & 23 & 82 \\
Primary endpoint & GOS-E; DRS and Mortality & GOS; Mortality and the modified FIM \\
\hline
\end{tabular}

GOS, glasgow outcome scale; GOS-E, glasgow outcome scale-extended; DRS, disability rating scale; FIM, functional independence measure. 
the injury-induced mRNA upregulation of interleukin 1 beta $(\mathrm{IL}-1 \beta)$ and tumor necrosis factor alpha $(\mathrm{TNFa})^{[35]}$. In the central nervous system, PROG inhibits TNFa-induced microglia activation, and decreases microglial expression of iNOS and secretion of IFNy, TGF- $\beta 2$, and NO, thus leading to a reduction in neural degeneration and apoptosis ${ }^{[36]}$. PROG treatment following TBI significantly decreased the levels of complement factors 3 and 5, macrophage-inducing factor-1, and CDs 24 and 74. Additionally, administering PROG shortly after CNS injury reduced both the migration and proliferation of immune cells ${ }^{[37]}$.

\section{Apoptosis}

PROG limits neuronal apoptosis by stabilizing the mitochondria. It can inhibit the activity of the mitochondrial-specific proapoptotic enzymes, such as cytochrome c, activated caspase-3, and the Bcl-2- associated death-promoter, and upregulate the anti-apoptotic versions of bcl-2 and the antiapoptotic protein $\mathrm{ERK}^{[38,39]}$. By modulating mitochondrial function, PROG can help to maintain cellular integrity and survival.

\section{Myelin repair and neurotrophic support}

PROG can promote both central and peripheral remyelination by increasing myelin production ${ }^{[40-42]}$. PROG treatment stimulates the synthesis of neurotrophic factors, such as brainderived neurotrophic factor (BDNF), at both the mRNA and protein levels ${ }^{[39,40]}$. Thus, PROG may prove to be a potential treatment for certain neurodegenerative disorders, such as multiple sclerosis ${ }^{[43]}$. Additionally, PROG increased the expression of nerve growth factor (NGF) in the brain, which correlates with post-TBI benefits including the attenuation of cholinergic cell death, promotion of axonal growth, prevention of axotomy-induced neuronal loss, and inhibition of neuronal apoptosis $^{[44]}$.

\section{Excitotoxicity}

PROG can attenuate neuronal excitotoxicity ${ }^{[45]}$ by blocking calcium channels $^{[46]}$ or by upregulating GABA, an inhibitory neurotransmitter in the $\mathrm{CNS}^{[47]}$. GABA mediated inhibition can then decrease the excessive injury induced excitotoxicity through the release of glutamate or other excitatory neurotransmitters ${ }^{[48]}$.

\section{Questions and prospects for phase III trials}

Although most preclinical studies and several promising clinical trials have reported benefits of PROG treatment, no beneficial effects were detected in three recent studies in which PROG was applied to animal models of spinal cord contusion injury, ischemic stroke or "moderate" unilateral contusion of the parietal cortex ${ }^{[49-51]}$. Despite the numerous studies that support the use of PROG in the treatment of brain injuries, some scientists still argue that the results of the PROG neuroprotection studies are too good to be true for a "gestational" hormonal agent that has been known for such a long time in the reproductive physiology and animal husbandry fields. Many researchers even believe that PROG has already failed the criteria of neuroprotection in its preclinical stage ${ }^{[52,53]}$.

Currently, all TBI clinical trials have failed in Phase III. It is reasonable to have some pessimism about the success of PROG, although the phase II clinical trials did show great promise. It should be emphasized that the Phase II trials reported above were small, single-centered clinical studies with a small number of recruited patients. The randomization of the placebo group:PROG group was 1:4 in Wright's ProTECT II trial. With a small number of placebo patients serving as controls, small outcome shifts in a few patients could result in huge, sometimes opposite, switches in the conclusions. More critically, no long-term assessment of the neurological outcome is available for these studies yet.

To expand the Phase II findings, two major Phase III trials were initiated in 2010. ProTECT III (progesterone for traumatic brain injury: experimental clinical treatment), initiated by Emory University and sponsored by the NIH, has been recruiting patients from 31 TBI clinical centers across the United States. SyNAPSe, initiated by BHR, a branch of Besins Pharma, in collaboration with the American Brain Injury Consortium (ABIC) and the European Brain Injury Consortium (EBIC), is another Phase III study investigating the efficacy and safety of progesterone in patients with severe traumatic brain injuries. SyNAPSe is a registration study and is designed to fulfill the requirements of the FDA for a future New Drug Application (Table 2). Two trials have passed their interim analyses for safety and futility and are proceeding according to the plan. No serious adverse events or reactions have been detected thus far. The SyNAPSe trial just recently completed the randomized enrollment of subjects in Aug 2013. Although the two trials can complement each other and may provide evidence for the use of PROG in the treatment of TBI, it should be noted that in the past 40 years, it is unusual that two phase III trials using the same medicine were conducted simultaneously in the TBI field, which again emphasizes the scientist's focus on PROG.

\section{Conclusion}

A large and rapidly growing body of preclinical studies suggested that PROG has neuroprotective properties in many brain injury models. The mechanism for PROG's neuroprotection clearly does not target a single aspect of the TBI cascade, instead it works through multiple mechanisms to enhance the repair of damage to nerve cells caused by CNS injury; for example, neurotrophic, anti-inflammatory, anti-excitotoxicity, anti-lipid peroxidation and anti-apoptotic properties and so on. PROG has been proven to be safe and has shown signs of efficacy in preliminary phase II clinical trials for TBI. The two Phase III multicenter trials that will validate the safety and efficacy of PROG for the treatment of TBI are currently being conducted. PROG could become the standard of care for the treatment of TBI if clear signs of efficacy are found in these two Phase III multicenter trials.

\section{Acknowledgements}

This review was supported by grants from the Key Medi- 
Table 2. Two randomized, double-blind, placebo-controlled phase III trials used to assess the efficacy and safety of progesterone in TBI patients.

\begin{tabular}{|c|c|c|}
\hline & ProTECT III & SyNAPSe \\
\hline Official title & $\begin{array}{l}\text { Progesterone for traumatic brain injury: } \\
\text { experimental clinical treatment }\end{array}$ & $\begin{array}{l}\text { A randomized, double-blind, placebo-controlled phase } 3 \\
\text { study to investigate the efficacy and safety of progesterone } \\
\text { in patients with severe traumatic brain injury }\end{array}$ \\
\hline Sponsor & Emory University & BHR pharma, LLC \\
\hline Collaborators & 31 Center of United States & PRA international and INC research \\
\hline Identifier & Nст00822900 & NCT01143064 \\
\hline Purpose & $\begin{array}{l}\text { Intravenous (IV) progesterone; within } 4 \mathrm{~h} \text { of } \\
\text { injury; a total of } 96 \mathrm{~h} \text {; moderate to severe TBI }\end{array}$ & $\begin{array}{l}\text { Intravenous (IV) progesterone; within } 8 \mathrm{~h} \text { of injury; a total of } \\
120 \mathrm{~h} \text {; severe TBI }\end{array}$ \\
\hline Primary outcome measures & GOS-E & GOS \\
\hline Secondary outcome measures & DRS; Mortality & SF-36; Mortality \\
\hline Estimated enrollment & 1140 & 1180 \\
\hline Study start date & March 2010 & June 2010 \\
\hline Estimated study completion date & June 2015 & August 2013 \\
\hline Ages eligible for study & 18 years and older & 16 years to 70 years \\
\hline Inclusion criteria & $\begin{array}{l}\text { Moderate to severe TBI (GCS } 12-4) \text {; blunt, } \\
\text { closed head injury; arrival <4 h from injury }\end{array}$ & $\begin{array}{l}\text { Severe TBI (GCS 8-3); at least one reactive pupil; arrival } \\
<8 \text { h from injury }\end{array}$ \\
\hline Health authority country & & $\begin{array}{l}\text { USA; Argentina; Austria; Belgium; China; Czech Republic; } \\
\text { Germany; Hungary; Israel; Italy; Netherlands; Romania; } \\
\text { Singapore; Spain; China Taiwan; United Kingdom and so on. }\end{array}$ \\
\hline Website & $\begin{array}{l}\text { http://clinicaltrials.gov/ct2/show/record/ } \\
\text { NCT00822900. }\end{array}$ & http://www.synapse-trial.com/ \\
\hline
\end{tabular}

GOS, glasgow outcome scale; GOS-E, glasgow outcome scale-extended; DRS, disability rating scale; SF-36, short form (36) health survey.

cal Research platform Program of Zhejiang Province, China (2013ZDA019) and the Key Science and Technology Development Program of Hangzhou, China (20100733Q07).

\section{References}

1 Bramlett HM, Dietrich WD. Progressive damage after brain and spinal cord injury: pathomechanisms and treatment strategies. Prog Brain Res 2007; 161: 125-41.

2 Stein DG. A clinical/translational perspective: can a developmental hormone play a role in the treatment of traumatic brain injury? Horm Behav 2013; 63: 291-300.

3 Guidelines for the management of severe head injury. Brain trauma foundation, American association of neurological surgeons, Joint Section on Neurotrauma and Critical Care. J Neurotrauma 1996; 13: 641-734.

4 Loane DJ, Faden Al. Neuroprotection for traumatic brain injury: translational challenges and emerging therapeutic strategies. Trends Pharmacol Sci 2010; 31: 596-604.

5 Roberts I, Yates D, Sandercock P, Farrell B, Wasserberg J, Lomas G, et al. Effect of intravenous corticosteroids on death within 14 days in 10008 adults with clinically significant head injury (MRC CRASH trial): randomised placebo-controlled trial. Lancet 2004; 364: 1321-8.

6 Gomes JA, Stevens RD, Lewin III JJ, Mirski MA, Bhardwaj A. Glucocorticoid therapy in neurologic critical care. Crit Care Med 2005; 33 : 1214-24.

7 Temkin NR, Anderson GD, Winn HR, Ellenbogen RG, Britz GW, Schuster J, et al. Magnesium sulfate for neuroprotection after traumatic brain injury: a randomised controlled trial. Lancet Neurol 2007; 6: 29-38.

8 Zafonte RD, Bagiella E, Ansel BM, Novack TA, Friedewald WT, Hesdorffer DC, et al. Effect of citicoline on functional and cognitive status among patients with traumatic brain injury: Citicoline Brain
Injury Treatment Trial (COBRIT). JAMA 2012; 308: 1993-2000.

9 Sullivan PG, Rabchevsky AG, Hicks RR, Gibson TR, Fletcher-Turner A, Scheff SW. Doseresponse curve and optimal dosing regimen of cyclosporine A after traumatic brain injury in rats. Neuroscience 2000; 101: 289-95.

10 Giroud M, Milan C, Beuriat P, Gras P, Essayagh E, Arveux P, et al. Incidence and survival rates during a two-year period of intracerebral and subarachnoid haemorrhages, cortical infarcts, lacunes and transient ischaemic attacks. the stroke registry of dijon: 1985-1989. Int J Epidemiol 1991; 20: 892-9.

11 Sacco RL, Boden-Albala B, Gan R, Chen X, Kargman DE, Shea S, et al. Stroke incidence among white, black, and hispanic residents of an urban community: The northern manhattan stroke study. Am J Epidemiol 1998; 147: 259-68.

12 Alkayed NJ, Murphy SJ, Traystman RJ, Hurn PD, Miller VM. Neuroprotective effects of female gonadal steroids in reproductively senescent female rats. Stroke 2000; 31: 161-8.

13 Carswell HV, Anderson NH, Clark JS, Graham D, Jeffs B, Dominiczak $A F$, et al. Genetic and gender influences on sensitivity to focal cerebral ischemia in the stroke-prone spontaneously hypertensive rat. Hypertension 1999; 33: 681-5.

14 Baulieu EE, Robel P. Neurosteroids: a new brain function? J Steroid Biochem Mol Biol 1990; 37: 395-403.

15 Schumacher M, Robel P, Baulieu EE. Development and regeneration of the nervous system: a role for neurosteroids. Dev Neurosci 1996; 18: 6-21.

16 Kato J, Hirata S, Nozawa A, Yamada-Mouri N. Gene expression of progesterone receptor isoforms in the rat brain. Horm Behav 1994; 28: 454-63.

17 Stein DG, Hoffman SW. Estrogen and progesterone as neuroprotective agents in the treatment of acute brain injuries. Pediatr Rehabil 2003; 6: 13-22.

18 Sayeed I, Stein DG. Progesterone as a neuroprotective factor in 
traumatic and ischemic brain injury. Prog Brain Res 2009; 175: 21937.

19 Xiao GM, Wei J, Wu ZH, Wang WM, Jiang QZ, Cheng J, et al. Clinical study on the therapeutic effects and mechanism of progesterone in the treatment for acute severe head injury. Zhonghua Wai Ke Za Zhi 2007; 45: 106-8. Chinese.

20 Xiao G, Wei J, Yan W, Wang W, Lu Z. Improved outcomes from the administration of progesterone for patients with acute severe traumatic brain injury: a randomized controlled trial. Crit Care 2008; 12: R61.

21 Wright DW, Kellermann AL, Hertzberg VS, Clark PL, Frankel M, Goldstein FC, et al. ProTECT: a randomized clinical trial of progesterone for acute traumatic brain injury. Ann Emerg Med 2007; 49: 391-402.

22 Anderson GD, Farin FM, Bammler TK, Beyer RP, Swan AA, Wilkerson $\mathrm{HW}$, et al. The effect of progesterone dose on gene expression after traumatic brain injury. J Neurotrauma 2011; 28: 1827-43.

23 Roof RL, Stein DG. Progesterone treatment attenuates brain edema following contusion injury in male and female rats. Restor Neurol Neurosci 1992; 4: 425-7.

24 Roof RL, Hall ED. Gender differences in acute CNS trauma and stroke: neuroprotective effects of estrogen and progesterone. J Neurotrauma 2000; 17: 367-88.

25 Wright DW, Bauer ME, Hoffman SW, Stein DG. Serum progesterone levels correlatewith decreased cerebral edema aftertraumatic brain injury in male rats. J Neurotrauma 2001; 18: 901-9.

26 Guo Q, Sayeed I, Baronne LM, Hoffman SW, Guennoun R, Stein DG. Progesterone administration modulates AQP4 expression and edema after traumatic brain injury in male rats. Exp Neurol 2006; 198: 469-78.

27 Limmroth V, Lee WS, Moskowitz MA. GABAA-receptor-mediated effects of progesterone, its ring-A-reduced metabolites and synthetic neuroactive steroids on neurogenic oedema in the rat meninges. $\mathrm{Br} J$ Pharmacol 1996; 117: 99-104.

28 Schumacher M, Guennoun R, Stein DG, De Nicola AF. Progesterone: therapeutic opportunities for neuroprotection and myelin repair. Pharmacol Ther 2007; 116: 77-107.

29 Roof RL, Hoffman SW, Stein DG. Progesterone protects against lipid peroxidation following traumatic brain injury in rats. Mol Chem Neuropathol 1997; 31: 1-11.

30 Moorthy K, Sharma D, Basir SF, Baquer NZ. Administration of estradiol and progesterone modulate the activities of antioxidant enzyme and aminotransferases in naturally menopausal rats. Exp Gerontol 2005; 40: 295-302.

31 Subramanian M, Pusphendran CK, Tarachand U, Devasagayam TP. Gestation confers temporary resistance to peroxidation in the maternal rat brain. Neurosci Lett 1993; 155: 151-4.

32 Pettus EH, Wright DW, Stein DG, Hoffman SW. Progesterone treatment inhibits the inflammatory agents that accompany traumatic brain injury. Brain Res 2005; 1049: 112-9.

33 Djebaili M, Hoffman SW, Stein DG. Allopregnanolone and progesterone decrease cell death and cognitive deficits after a contusion of the rat pre-frontal cortex. Neuroscience 2004; 123: 349-59.

34 VanLandingham JW, Cekic M, Cutler S, Hoffman SW, Stein DG. Neurosteroids reduce inflammation after TBI through CD55 induction. Neurosci Lett 2007; 425: 94-8.

35 He J, Evans CO, Hoffman SW, Oyesiku NM, Stein DG. Progesterone and allopregnanolone reduce inflammatory cytokines after traumatic brain injury. Exp Neurol 2004; 189: 404-12.

36 Drew PD, Chavis JA. Female sex steroids: effects upon microglial cell activation. J Neuroimmunol 2000; 111: 77-85.

37 Nilsen J, Brinton RD. Impact of progestins on estradiol potentiation of the glutamate calcium response. NeuroReport 2002; 13: 825-30.
38 Djebaili M, Hoffman SW, Stein DG. Allopregnanolone and progesterone decrease cell death and cognitive deficits after a contusion of the rat pre-frontal cortex. Neuroscience 2004; 123: 349-59.

39 Stein DG, Wright DW. Progesterone in the clinical treatment of acute traumatic brain injury. Expert Opin Investig Drugs 2010; 19: 847-57.

40 De Nicola AF, Gonzalez SL, Labombarda F, Deniselle MC, Garay L, Guennoun R, et al. Progesterone treatment of spinal cord injury: effects on receptors, neurotrophins, and myelination. J Mol Neurosci 2006; 28: 3-15.

41 Gonzalez SL, Labombarda F, Gonzalez Deniselle MC, Guennoun R, Schumacher M, De Nicola AF. Progesterone up-regulates neuronal brain-derived neurotrophic factor expression in the injured spinal cord. Neuroscience 2004; 125: 605-14.

42 Labombarda F, Gonzalez S, Gonzalez Deniselle MC, Garay L, Guennoun R, Schumacher M, et al. Progesterone increases the expression of myelin basic protein and the number of cells showing NG2 immunostaining in the lesioned spinal cord. J Neurotrauma 2006; 23: 181-92.

43 Schumacher M, Guennoun R, Robert F, Carelli C, Gago N, Ghoumari A, et al. Local synthesis and dual actions of progesterone in the nervous system: neuroprotection and myelination. Growth Horm IGF Res 2004; 14: S18-S33.

44 Cekic M, Johnson SJ, Bhatt VH, Stein DG. Progesterone treatment alters neurotrophin/proneurotrophin balance and receptor expression in rats with traumatic brain injury. Restor Neurol Neurosci 2012; 28 : $1-12$.

45 Smith SS. Progesterone administration attenuates excitatory amino acid responses of cerebellar Purkinje cells. Neuroscience 1991; 42: 309-20.

46 Luoma JI, Kelley BG, Mermelstein PG. Progesterone inhibition of voltage-gated calcium channels is a potential neuroprotective mechanism against excitotoxicity. Steroids 2011; 76: 845-55.

47 Shen $\mathrm{H}$, Gong QH, Yuan M, Smith SS. Short-term steroid treatment increases delta GABAA receptor subunit expression in rat CA1 hippocampus: pharmacological and behavioral effects. Neuropharmacology 2005; 49: 573-86.

48 Brann DW, Zamorano PL, De Sevilla L, Mahesh VB. Expression of glutamate receptor subunits in the hypothalamus of the female rat during the afternoon of the proestrous luteinizing hormone surge and effects of antiprogestin treatment and aging. Neuroendocrinology 2005; 81: 120-8.

49 Fee DB, Swartz KR, Joy KM, Roberts KN, Scheff NN, Scheff SW. Effects of progesterone on experimental spinal cord injury. Brain Res 2007; 1137: 146-52.

50 Toung TJ, Chen TY, Littleton-Kearney MT, Hurn PD, Murphy SJ. Effects of combined estrogen and progesterone on brain infarction in reproductively senescent female rats. J Cereb Blood Flow Metab 2004; 24: 1160-6.

51 Gilmer LK, Roberts KN, Scheff SW. Efficacy of progesterone following a moderate unilateral cortical contusion injury. J Neurotrauma 2008; 25: 593-602.

52 Stein DG. Progesterone in the treatment of acute traumatic brain injury: a clinical perspective and update. Neuroscience 2011; 191 : 101-6.

53 Stoica B, Byrnes K, Faden Al. Multifunctional drug treatment in neurotrauma. Neurotherapeutics 2009; 6: 14-27.

(a) This work is licensed under the Creative Commons Attribution-NonCommercial-Share Alike 3.0 Unported License. To view a copy of this license, visit http://creativecommons.org/licenses/by-nc-sa/3.0/ 